\title{
RESPIRABLE DUST DOSIMETER (RDD) FOR PERSONAL DUST EXPOSURE ASSESSMENT-A LABORATORY INVESTIGATION
}

\author{
BK Belle \\ Anglo Technical Division, Johannesburg, South Africa
}

\begin{abstract}
The search for an improved or alternative instrument that will be able to measure occupational exposure more accurately and more reliably is continuing. Several developments have been taken place overseas with regard to real-time monitoring instruments for personal exposure assessment. In the light of these developments, the respirable dust dosimeter (RDD) described by Volkwein et al. (2000) appeared to be a promising development. RDD measures the dust exposure in differential pressure units ( $\mathrm{mm}$ of $\mathrm{Hg}$ ) indicating that it is a surrogate for the mass of dust on a gravimetric filter. This paper describes the results of an evaluation on the RDD under laboratory conditions before evaluating them in underground mines for personal dust exposure assessment.
\end{abstract}

The laboratory study has shown that there is a potential for the RDD sampler as a screening tool for engineering sample purposes. However, RDD was comparatively expensive during the research exercise costing approximately 16 UK Pounds per RDD tube. Further, there was no well-accepted procedure on collect dust samples using RDD tube for further quartz analysis. Finally, due to its reasonable estimates of real-time cumulative shift dust exposure, and its small lightweight package, the RDD was further recommended for personal sampling purposes in the South African mines.

\section{INTRODUCTION*}

*Views expressed are solely of the author and do not reflect any organisation.

Monitoring of dust in the mines is an important task in terms of Section 12.2 and 12.3 of the Mine Health and Safety Act (MHSA) of 1996 and therefore requires reliable dust measuring instruments. Information on routine worker dust-exposure levels using reliable dust monitors can assist both workers' and operators' awareness of the need to protect their occupational respiratory health.

The search for an improved or alternative instrument to measure occupational exposure more accurately and more reliably is continuing. Several developments have been taken place overseas with regard to near-real-time monitoring instruments for personal exposure assessment. In the light of these developments, the respirable dust dosimeter (RDD) developed by the National Institute of Occupational Safety and Health (NIOSH) appeared to be a promising instrument for personal dust exposure measurement. This paper describes the laboratory investigations into the newly developed RDD tube for personal exposure assessment in South African mines.

In order for the introduction of the new dust-monitoring instruments for personal sampling in underground mines, they were required to meet the following criteria:

- Intrinsically safe (IS) for use in South African underground mines.

- Sample according the size-selective criteria (ISO/CEN/ACGIH curve) at specified flow rates.

- Meet the $\pm 25 \%$ NIOSH or CEN $50 \%$ accuracy criterion.

- Preferably use quick analytical procedure to the current weighing method.

- Give real-time dust concentration values, cumulative shift exposure and sampling time.

- Robust enough to withstand the harsh conditions prevailing in South African mines.

- Cost-effective and be able to collect dust samples for further quartz analysis.

- Compact and portable for personal sampling.

Respirable dust dosimeter (RDD)

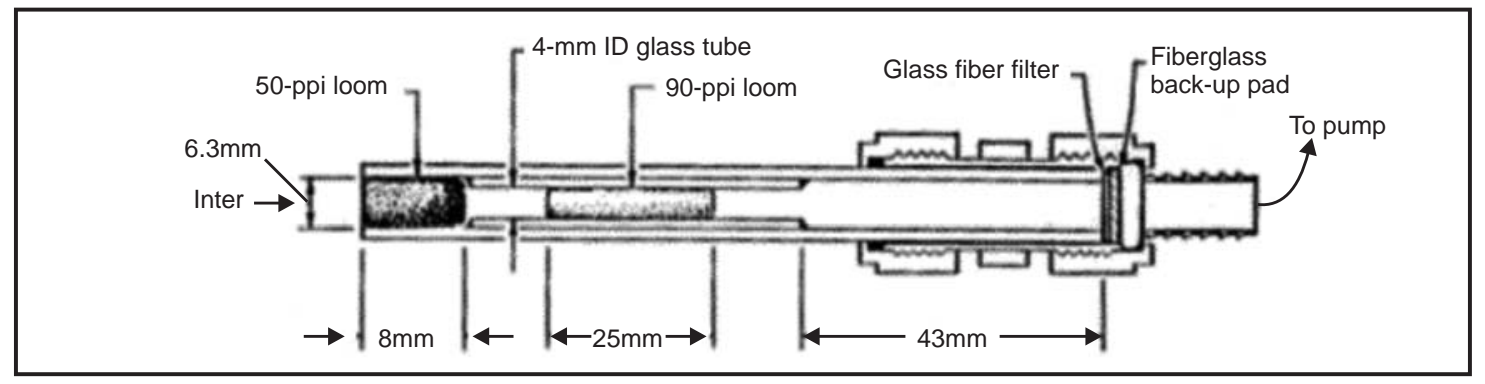

Figure 1. Cross-section of the Respirable Dust Dosimeter (RDD) (Source: Volkwein et al., 2000) 
The laboratory performance of a detector tube made from glass and brass that had good correlation between differential pressure and mass has been described by the NIOSH researchers (Volkwein et al., 2000; Page et al., 2000).

A commercially available, intrinsically safe, low flow rate air-sampling pump with integral pressure transducer is used to monitor the pressure increase with mass loading. Figure 1 shows parts of a complete RDD. The increasing pressure differential across the filter created by accumulation of dust is measured and correlated with respirable mass.

Dust enters the inlet of the detector tube, as illustrated in Figure 1, through a $6.3 \mathrm{~mm}$ diameter by $8.0 \mathrm{~mm}$ length of polyurethane open-cell foam with a density of $50 \mathrm{ppi}$ (pores per inch). This segment filters out oversized non-respirable dust and protects the main classifier from being plugged with oversize material. The tube narrows to a $4.0 \mathrm{~mm}$ diameter section, which contains a $25.0 \mathrm{~mm}$ length of $90 \mathrm{ppi}$ open-cell urethane foam; this collects the non- respirable dust and passes the respirable fraction of the dust (Volkwein et al., 2000).

The flow path of the classified respirable fraction of the dust gradually expands in the detector tube to $6.3 \mathrm{~mm}$ diameter, and travels $55.0 \mathrm{~mm}$ to uniformly deposit onto the collection filter. The respirable dust deposits onto an $8.0 \mathrm{~mm}$ diameter fluorocarbon-coated glass fibre filter, supported by a porous fibre back-up pad. The respirable classification section and the filter holder are made of conductive plastic, which is ultrasonically welded together. At the recommended flow rate of $250 \mathrm{ml} / \mathrm{min}$, the RDD samples according to the new ISO/CEN/ACGIH respirable curve, with a D50 of 4 microns.

\section{Previous evaluations on RDD}

Previous work on the RDD has been carried out in the USA and UK coal mines. In the USA, the measurements using the RDD and the Dorr-Oliver cyclone (operated at a flow rate of $1.7 \mathrm{~L} / \mathrm{min}$ according to the ISO/CEN/ACGIH curve) were compared in both state-of-the-art laboratory and field trials. The averages of the combined set of laboratory and mine data showed a strong predictive linear relationship (95\%) between the RDD and the gravimetric sampler data. The studies have indicated that the RDD reading (in $\mathrm{mm}$ of $\mathrm{Hg}$ ) is a good surrogate for the mass of dust on a gravimetric filter, and can be used to approximate the cumulative dust exposure, calculated as either a mass or a time-weighted concentration (Ramani et al., 2001).

Similarly, in an another study (Kenny, 2001) by the Health and Safety Laboratory (HSL), various dust-monitoring instruments viz., MRE 113a, CIP10, RDD and Electret were evaluated for personal and area monitoring purposes in the UK mines. The study concluded that the RDD is a less precise instrument. However, it was reported that the RDD could be used for screening or background measurements of respirable dust, especially for assessment purposes.

\section{LABORATORY EVALUATIONS- POLLEY DUST DUCT}

The Polley dust duct (Figure 2) is a dust chamber in which a steady concentration can be established and low concentrations can be maintained for long periods.

Currently, the duct is located in the Air Quality section of the National Council of Occupational Health $(\mathrm{NCOH})$ in Johannesburg. The duct was designed and built in Germany by Dr. Hans Polley. This is the only duct available outside Germany. Its unique feature is that a near-desired dust concentration of any dust type can be established (using only dust briquettes) and maintained in the duct (Du Toit, 2001).

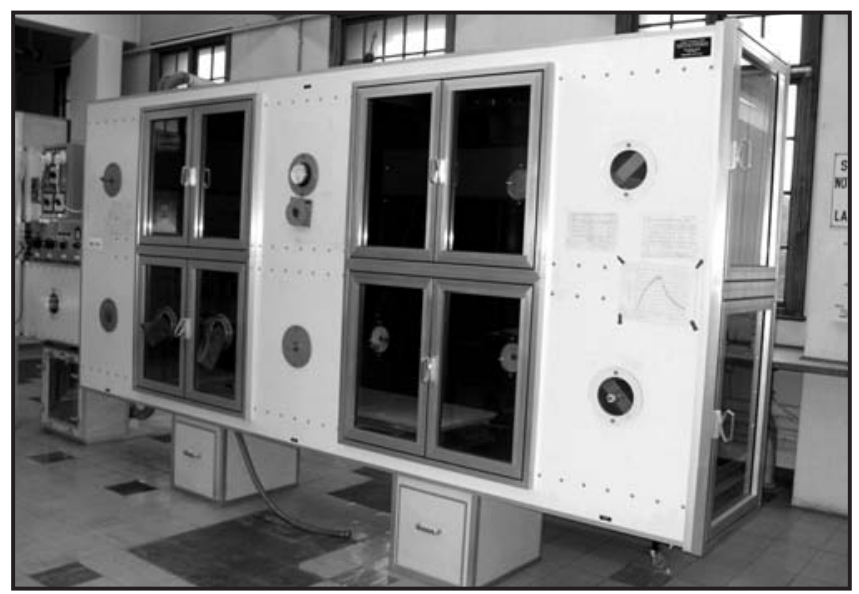

Figure 2: $\quad$ Photo of the Polley dust duct

\section{Description of Polley dust duct}

The Polley dust duct consists of a closed-circuit duct, two dust generators (Figure 3) and two fans (Du Toit et al., 1973). The instantaneous dust concentration levels can only be recorded with gravimetric samplers in conjunction with real-time monitors.

The closed-circuit duct consists of two sections: a horizontal section and a vertical section. The horizontal section is the main section and measures $7.0 \mathrm{~m}$ long by $2.0 \mathrm{~m}$ high by $0.7 \mathrm{~m}$ wide. The air flows along the horizontal channel into and along the top half of the large horizontal section. It returns along the bottom half of the large horizontal section through a flow-straightening section (Figure 3) and flows along the lower, small horizontal channel into and upwards in the vertical section to close the circuit. The duct also has other auxiliary parts such as time relays, two fans to circulate the air and a third to exhaust the dust-laden air through a filter to atmosphere, and a dust briquette press. 


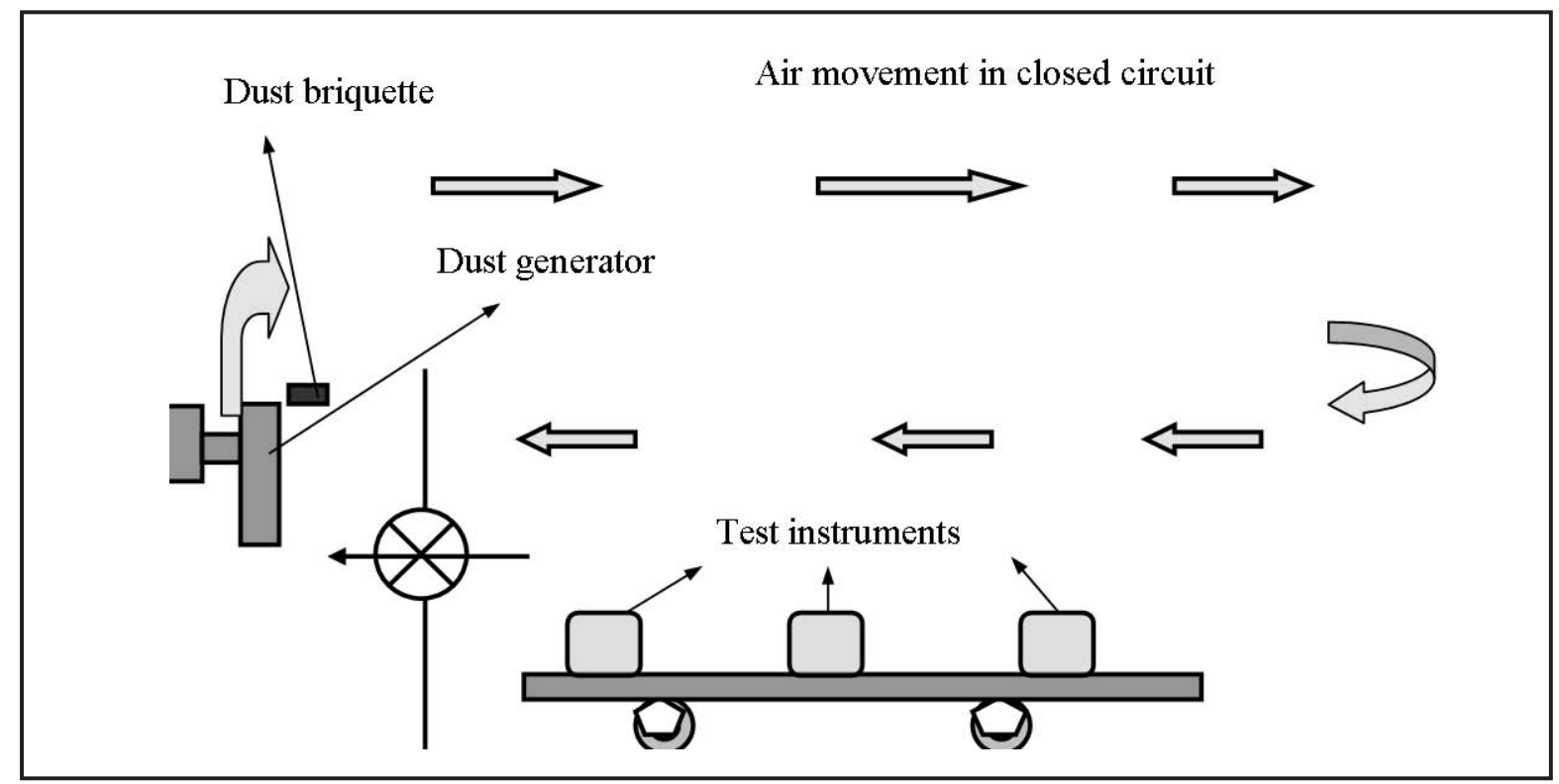

Figure 3: $\quad$ Line diagram of the Polley dust duct operation

\section{Feed dust briquette}

Standard sandstone briquettes and laboratory-prepared bituminous coal dust briquettes were used in this study. The coal dust used was that prepared and fractionated to obtain fine dust for experiments. For the analysis of the size distribution of the coal dust used for the briquette, a Fritsch Particle Size Analyser was used. The average briquette dust sample contained $50 \%$ of particles with a size of $22.0 \mu \mathrm{m}$. Both the coal dust and the prepared coal dust briquettes were stored in airtight bags to prevent oxidation. The quartz content of the sandstone briquette dust, analysed using Xray diffraction technique. The average quartz content of three respirable sandstone briquette dust samples was 50.63 $\%$.

\section{Laboratory Sampling Plan}

The dust concentration level inside the chamber was determined by the gravimetric sampling method, according to the established DME procedures (DME, 1997). The Polley duct did not have the facility to determine the realtime size distribution of the airborne dust inside the chamber. For each laboratory test, the evaluation instrument and reference samplers were positioned side by side inside the lower chamber of the Polley duct, with their monitoring inlets as close together as possible (Figure 4). Sampler inlets faced the direction of the airflow in order to avoid the effect of nozzle inlet orientation on sampler performance. For the laboratory study, the identified monitoring instruments were exposed to two types of dust, viz. coal and sandstone briquette dust.

\section{Reference Samplers}

The locally manufactured and widely used gravimetric sampler (GME \# 05) operated at $2.2 \mathrm{~L} / \mathrm{min}$ in accordance with the new ISO/ACGIH/CEN curve with a D50 of 4 microns (Kenny et al., 1998) was used as a reference sampler for this study. Measurement of the size-selection characteristics of the South African cyclone, as tested in the UK (Kenny, Baldwin and Maynand, 1998), confirmed that they are similar to those of the Higgins-Dewell (HD) designs.

Similarly, Dorr-Oliver type sampler (as used in the US studies) was also used in the tests. The study by Liden and Kenny (1993) suggests that operating Dorr-Oliver $10 \mathrm{~mm}$ cyclone at a flow rate of $1.7 \mathrm{~L} / \mathrm{min}$ is actually most appropriate for sampling according to the new ISO/CEN/ACGIH definition of respirable dust with a D50 of 4 microns. Figure 4 shows a typical side-by-side positioning of samplers in the test chamber.

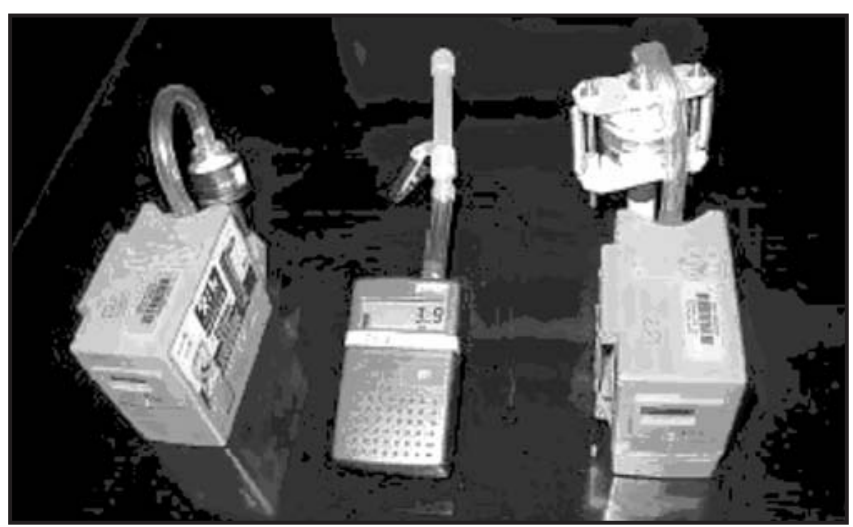

Figure 4: $\quad$ Side-by-Side position of SA sampler (left), $R D D$ (middle) and D-O sampler (right) 
Experimental Procedure

The following procedure was followed during the laboratory studies:

- Before the start of the experiment, the chamber was cleaned of all the dust adhering to the chamber. Clean air was passed through, using an exhaust fan, for more than five minutes to create a continuous flow of fresh air prior to the introduction of coal or sandstone dust.

- The generator was then switched on and the dust was fed into the chamber by switching on the dust generators. An equilibrium dust concentration was reached in approximately 10 to 15 minutes.]

- Before the start of the experiment, the pumps of all the identified monitoring instruments were calibrated. A Gillibrator primary standard flow meter was used to establish the required flow rate for each dust monitor, using an equivalent pressure restriction of the cyclone and filter assembly.

- The evaluation instruments were positioned on the sampling table at various positions. Throughout the experiment, the air pumps and the condition of the sampling train were monitored for operation.

- The sampling time was chosen in such a way that enough dust was collected on the filter (approximately 60 minutes).

- The dust monitors were operated according to the required flow rate so that they will sample according to the new CEN/ACGIH/ISO size-selective curve.

- After sampling, the air pumps and RDD were turned off and time noted down. The gravimetric dust samples were removed from the samplers for mass determination. Filters from the samplers were weighed on an analytical electronic balance capable of an accuracy of $0.0001 \mathrm{mg}$.

- At the conclusion of the test, a PC was used to read the data from the RDD real-time monitoring instruments.

- After completion of each test, the sampling units (dust monitors) were cleaned, new filters were installed and they were prepared for the next test.

- After sampling, the dust generators were turned off. When all the experiments with a particular dust type have been completed, the dust chamber was cleaned.

\section{Results and Discussions}

During the laboratory trials, it was initially assumed that the variability from tube to tube was negligible and it was expected that the SKC dosimeter pump would calculate the differential pressure directly. However, the older-version dosimeter pump (which was used in the test) did not have the feature that would measure the direct pressure drop with increase in mass loading of respirable dust. Therefore, the variability from tube to tube was incorporated into the laboratory results.

In order to determine the variability, the RDD tubes were tested in a clean weighing room. Each of the RDD tubes were ran for 30 seconds and the initial pressures were measured. The summary statistics of the initial pressures measured for the RDD tubes is shown in Table 1. The average of the initial RDD tube pressures was subtracted from the final laboratory-measured pressure data.

Table 1. Summary statistics of initial RDD pressures.

\begin{tabular}{l|l}
\hline $\begin{array}{l}\text { Statistic } \\
\text { Pressures }\end{array}$ & RDD Initial \\
\hline Mean & 3.507 \\
\hline Variance & 0.052 \\
\hline Median & 3.482 \\
\hline Std. dev. & 0.227 \\
\hline Minimum & 2.786 \\
\hline Maximum & 4.571 \\
\hline Size of sample & 168 \\
\hline
\end{tabular}

Figure 5 shows the histogram of the initial pressures of the RDD tubes. From the above table and the distribution plot (Figure 5) it can be seen that the initial pressures of the RDD tubes were normal, with minimum and maximum initial measured pressures of $2.786 \mathrm{~mm}$ of $\mathrm{Hg}$ and $4.571 \mathrm{~mm}$ of $\mathrm{Hg}$ respectively. The mean initial pressure for the 168 tubes measured was $3.507 \mathrm{~mm}$ of $\mathrm{Hg}$.

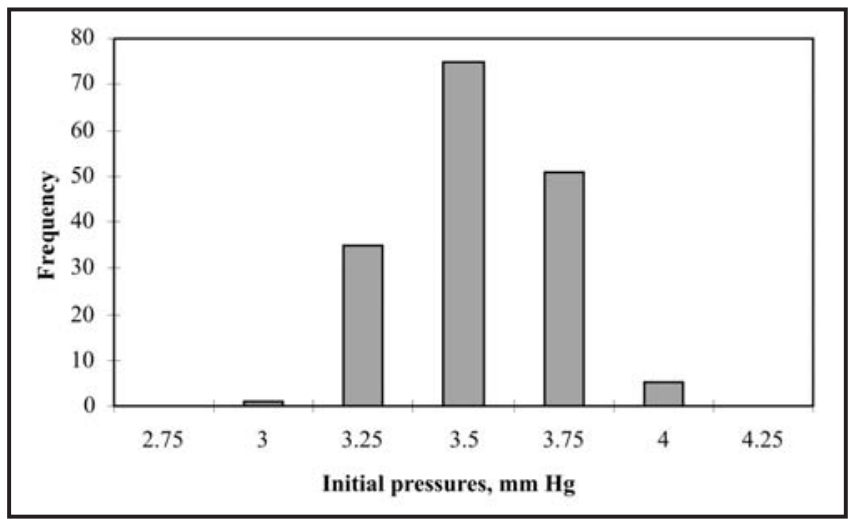

Figure 5: $\quad$ Histogram of the initial pressures of RDD

The RDD pressures and the mass of dust collected on the filter using SA cyclone and the DO cyclone are plotted and discussed below. The scatter plot and the least-squares regression of the gravimetric sample respirable mass versus the dosimeter differential pressures for the side-by-side comparisons with both coal and sandstone dust are shown in Figures 6 and 7 respectively.

Overall, the plots show the linear relationship between the RDD and SA samplers. The scatter was wide for all levels of respirable coal dust, but it was narrow for the respirable sandstone dust, with a power relationship giving the best fit. It was also recognized that when two samplers (the SA 
sampler and the Dorr-Oliver sampler) were operated according to the same sizeselective curve, there was a significant difference in the measured respirable dust concentrations for both coal and sandstone dust.

The linear relationship between the RDD and SA samplers was plotted for the combined dust data and it was found that an average relationship between two samplers (Figure 8). The combined data for the plot of both coal and sandstone dust indicate a good coefficient of determination. From the laboratory trials, it can be concluded that the relationship between the RDD and SA gravimetric samplers was average. The probable differences could be attributed to the variations in initial pressure between the RDD tubes. In conclusion, the laboratory study showed the potential of the RDD sampler as a screening tool for engineering sample purposes.

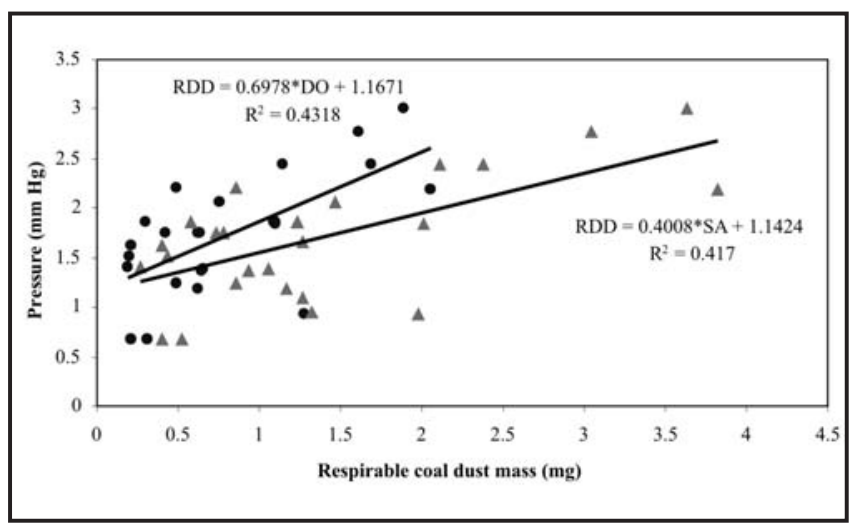

Figure 6: Relationship between RDD tube and gravimetric samplers for coal dust

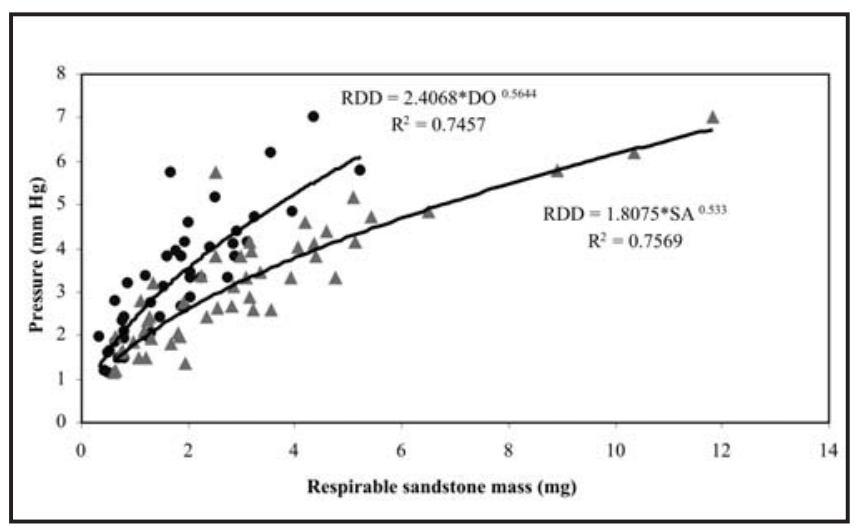

Figure 7: Relationship between RDD tube and gravimetric samplers for sandstone dust

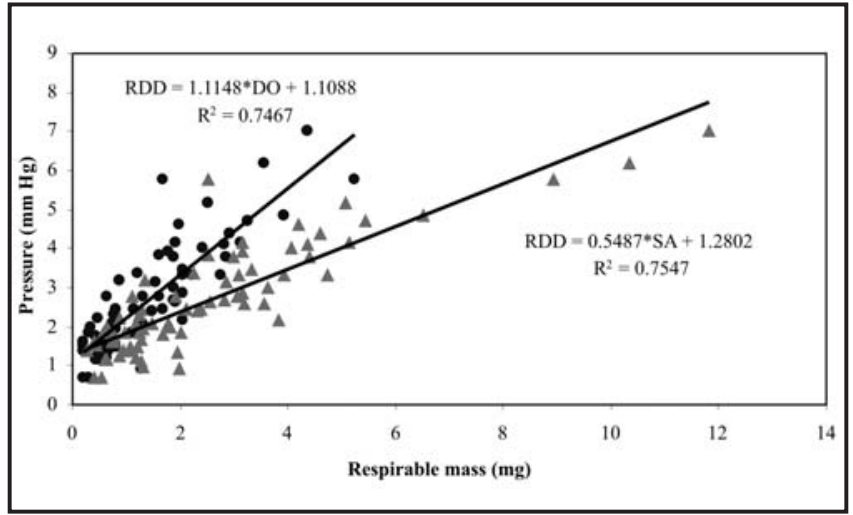

Figure 8: $\quad$ Combined coal and sandstone dust data on the relationship between $R D D$ tube and gravimetric samplers

\section{Conclusions}

The following conclusions can be drawn from the laboratory evaluation of the RDD:

- There is a significant difference in the measured dust levels between SA sampler and Dorr-Oliver sampler when operated according to the same size-selective curve (ACGIH/ISO/CEN).

- NIOSH RDD can be potentially used as a screening tool and there was an average linear relationship between increase in pressure of RDD and respirable mass collected on the gravimetric filter.

- The RDD real-time dust monitor was easy to use and it is envisaged that its application as a personal sampler under harsh mining is very high. Further, the RDD is not recommended for use in an environment where diesel particulate matter (DPM) and moisture is present (Volkwein et al., 2000). Also, there is no confirmed procedure for evaluating the quartz content of the RDD foam dust sample.

Finally, the RDD tube approach to personal dust monitoring offers many advantages and meets the following requirements for use in South African mines:• RDD is intrinsically safe (IS), compact, portable and robust to withstand the harsh conditions prevailing in South African mines for personal sampling.

- RDD samples according to the size-selective criteria (ISO/CEN/ACGIH curve) at specified flow rates (Volkwein et al., 2000).

- RDD uses quick analytical procedure to the weighing method that is currently used.

- RDD gives real-time dust levels and cumulative shift exposure in $\mathrm{mm}$ of $\mathrm{Hg}$ and sampling time.

However, RDD was comparatively expensive during this research exercise costing approximately 16 UK Pounds per tube. Also, there was no well-accepted procedure on collect 
dust samples using RDD tube for further quartz analysis. Finally, due to its reasonable estimates of real-time cumulative shift dust exposure, and its small lightweight package, the RDD was recommended for further evaluations for personal sampling purposes in the South African mines.

\section{Acknowledgements}

The research findings presented in this paper was part of a SIMRAC sponsored project Health 704 carried out by the Author. The support of CSIR-Miningtek is appreciated. Gratitude is expressed to John Volkwein of NIOSH for the advice in data handling of the RDD during initial stages of the research work.

\section{References}

Biffi, M., Belle, B.K. and Unsted, D., 2000, Proposed rational criteria for routine dust sampling of respirable dust in South African mines. HEALTH 604b, SIMRAC Research Report, South Africa, 110 p.

Du Toit, R.S.J., 2001, Personal Communications, NCOH, South Africa.

Du Toit, R.S.J., Murray, N.M. and Owen, T.B., 1973, A steady concentration dust duct. Journal of the Mine Ventilation Society of South Africa.
DME. 1997. Guidelines for the gravimetric sampling of airborne particulate for risk assessment in terms of the occupational diseases in mines and works act \# 78 of 1978, Parent Doc. 3rdEd. SA.

Kenny, L. Baldwin, P.E.J. and Maynard, A. D. 1998. Respirable dust sampling at very high concentrations, HSL, UK.

Kenny, L.C., 2001, Improved dust sampling methods for mines. HSE Report, IR/A/98/05, UK.

Page, S.J., Volkwein J.C., Baron P.A. and Deye G.J., 2000, Particulate penetration of porous foam as a low flow rate respirable dust size classifier. Appl. Occup. \& Environ. Hyg., 15(7): 561-568.

Ramani R.V., Mutmansky, J.M., He, H., Volkwein J.C., Marple, V.A., Olson, B. and Luna, P., 2001, A comparative evaluation of the differential pressure-based dust detector with the personal gravimetric respirable dust sampler in underground coal mines. 7th International Mine Ventilation Congress, Krakow, Poland.

Volkwein, J.C., Schoeneman, A.L. and Page, S.J., 2000, Laboratory evaluation of pressure differential based respirable dust detector tube. Appl. Occup. \& Environ. Hyg., 15(1): 158-164. 